Here we have used (2.5).

I wish to thank the referee for some improvements in $\$ 2$ of this paper.

\title{
REFERENCES
}

1. B. Gordon, Two new representations of the partition function, Proc. Amer. Math. Soc. 13 (1962), 869-873.

2. G. H. Hardy and E. M. Wright, An introduction to the theory of numbers, 4th ed., Oxford Univ. Press, Oxford, 1960.

3. J. J. Sylvester, $A$ constructive theory of partitions, Amer. J. Math. 5 (1882), 251-330.

los Angeles, California

\section{MULTIPLE TRANSITIVITY OF PRIMITIVE PERMUTATION GROUPS}

\section{A. MACHTINGER}

Introduction. We wish to consider two theorems on permutation groups which are a generalization of a two-part theorem found in $[1$, pp. 66-67], a theorem concerned with a permutation group on a finite set. We shall remove the restriction of finiteness.

The following notation and definitions will be used in the discussion:

If $Y$ is a set, then $|Y|$ denotes the cardinal number of $Y$. If $g$ is a mapping of $X$ into $Z$ and $x \in X$, then $x g$ denotes the image of $x$ under $g$. If $Y \subseteq X$, then $(Y) g$ denotes the image of $Y$ under $g$. If $G$ is a group and $H \leqq G$ and $g \in G$, then $H^{o}=g^{-1} H g$. A permutation group $G$ on a set $X$ is said to be $r$-ply (or $r$-fold) transitive on $Y \subseteq X$ (where $r$ is a nonzero cardinal number and $|Y| \geqq r$ ), if (i) for every pair of subsets $A$ and $B$ of $Y$ of cardinal number $r$ and for any one-to-one map $f$ of $A$ onto $B$, there exists $g \in G$ such that $\left.g\right|_{A}=f$ (where $\left.g\right|_{A}$ is the restriction of $g$ to $A$ ), and if (ii) $(Y) g=Y$ for every $g \in G$. A 1 -fold transitive group on $Y \subseteq X$ is called transitive on $Y$.

A permutation group $G \neq 1$ on a set $X$ is said to be imprimitive on $Y \subseteq X$ if: (i) $Y$ can be written as a disjoint union of two or more nonvoid sets $\left\{S_{\alpha}\right\}_{\alpha \in \mathfrak{r}}$, (ii) for at least one $\alpha \in \mathfrak{A},\left|S_{\alpha}\right| \geqq 2$ and (iii) given $g \in G$ and $\alpha \in \mathscr{A}$, then $\left(S_{\alpha}\right) g=S_{\beta}$ for some $\beta \in \mathfrak{A}$. The sets $S_{\alpha}$, $\alpha \in \mathfrak{A}$, are called imprimitivity sets of $G$ on $Y$. If $G$ is not imprimitive on $Y$, then $G$ is called primitive on $Y$.

Received by the editors August 19, 1963. 
The theorem which we shall generalize is:

Let $G$ be a permutation group on $n$ letters which is primitive, and let $H$ be a transitive subgroup of $G$ on $m$ letters (where $m \geqq 2$ ) fixing the remaining $n-m$ letters. Then (1) if $H$ is primitive, $G$ is $(n-m+1)$ fold transitive; (2) in any event, $G$ is doubly transitive.

We shall prove:

THEOREM 1. Let $G$ be a primitive permutation group on $X$ where $|X| \geqq 3$. Suppose that there exists a subgroup $H \leqq G$ which (i) is primitive and $r$-fold transitive on a subset $Y \subseteq X$ where $|Y| \geqq 2$ and where $N=|X \backslash Y|$ is a positive integer, and which (ii) fixes the elements of $X \backslash Y$ (i.e., if $x \in X \backslash Y, g \in H$, then $x g=x$ ). Then $G$ is $(N+r)$-fold transitive on $X$.

We shall not consider the case where $N$ is an infinite cardinal number.

Theorem 2. A permutation group $G$ on a set $X$ where $|X| \geqq 3$ is doubly transitive on $X$ if and only if (i) $G$ is primitive, (ii) there exists a subgroup $H \leqq G$ which is transitive on some $Y \subseteq X$, where $|Y| \geqq 2$ and $X \backslash Y$ is finite and nonvoid, and (iii) $H$ fixes the elements of $X \backslash Y$.

It should be noted that: (1) if $G$ is $m$-ply transitive on $X$, where $m=r+N, r \geqq 1$ and $N \geqq 1$, then there exists a subgroup $H \leqq G$, which is $r$-fold transitive on $Y \subseteq X$, where $|X \backslash Y|=N$ and $H$ fixes the elements of $X \backslash Y$; (2) if $G$ is $m$-fold transitive on $X$ where $m \geqq 2$, then $G$ is primitive [1, pp. 56 and 66]. Hence we have the following partial converse to Theorem 1 : If $G$ is an $(N+r)$-fold transitive permutation group on $X$, where $N \geqq 1, r \geqq 2$, then $G$ is primitive, and there is a subgroup $H \leqq G$, which (i) is primitive and $r$-fold transitive on some $Y \subseteq X$, where $|Y| \geqq 2$ and $N=|X \backslash Y|$, and which (ii) fixes the elements of $X \backslash Y$. It is also true that a nontrivial primitive permutation group $G$ on a set $X$ is transitive on $X[1$, p. 64].

We shall need the following propositions:

Proposition 1. Let $G$ be a permutation group on $X$ and let $H \leqq G$. (1) If $H$ is r-fold transitive on $Y \subseteq X$ and fixes the elements of $X \backslash Y$, then $H^{\circ}$ (where $g \in G$ ) is r-fold transitive on $(Y) g$ and fixes $(X \backslash Y) g$ $=X \backslash(Y) g$. (2) If $H$ is primitive on $Y \subseteq X$, then $H^{\circ}$ is primitive on $(Y) g$ for every $g \in G$.

Proposition 2. Let $G$ be a permutation group on $X$, and let $H_{1}$, $H_{2} \leqq G$ such that (i) $H_{j}$ is transitive on $Y_{j} \subseteq X$ and fixes $X \backslash Y_{j}, j=1,2$, and (ii) $Y_{1} \cap Y_{2} \neq \varnothing$. Then $H=\left\{H_{1}, H_{2}\right\}$ is transitive on $Y=Y_{1} \cup Y_{2}$ and fixes $X \backslash Y$. 
Proposition 3. Let $G$ be an s-fold transitive permutation group on $X$, and suppose that there is a subgroup $H \leqq G$ such that (i) $H$ is r-fold transitive on $Y \subseteq X$, (ii) $H$ fixes $X \backslash Y$ and (iii) $s=|X \backslash Y|$. Then $G$ is $(r+s)$-fold transitive on $X$.

The proofs of these propositions are simple [1, pp. 56, 66-67].

Proposition 4. Let $Y \subseteq X$ such that $X \backslash Y$ is finite, and let $f$ be a permutation of $X$. Let $C=Y \cap(Y) f, A=Y \backslash C$ and $B=(Y) f \backslash C$. Then (i) $A, B$ and $C$ are pairwise disjoint, (ii) $A$ and $B$ are finite with $|A|=|B| \leqq|X \backslash Y|$ and (iii) $Y=C \cup A,(Y) f=C \cup B$.

Proof. Since $(X \backslash Y) f=X \backslash(Y) f, \quad|X \backslash Y|=|X \backslash(Y) f|$. Let $W=Y \cup(Y) f$. Then $X \backslash Y=(X \backslash W) \cup((Y) f \backslash C)$ and $X \backslash(Y) f=(X \backslash W)$ $\cup(Y \backslash C)$ where $(X \backslash W) \cap((Y) f \backslash C)=\varnothing$ and $(X \backslash W) \cap(Y \backslash C)=\varnothing$. Hence $|X \backslash Y|=|X \backslash W|+|(Y) f \backslash C|$ and $|X \backslash(Y) f|=|X \backslash W|$ $+|Y \backslash C|$. From the finiteness of the above numbers, we infer $|A|=|B| \leqq|X \backslash Y|$.

Proof of Theorem 1. We use induction on $N$. If $N=1$, the desired result follows for all cardinal numbers $r \geqq 1$ by Proposition 3. Suppose that the result is true for $N_{0} \geqq 1$ and for all $r \geqq 1$, and assume that $N=N_{0}+1$. We wish to show the existence of an $H_{0} \leqq G$ such that $H_{0}$ is $(r+1)$-fold transitive on some $Y_{0} \subseteq X$ where $\left|Y_{0}\right| \geqq 2, N_{0}=\left|X \backslash Y_{0}\right|$ and $H_{0}$ fixes $X \backslash Y_{0}$ (then $H_{0}$ will be at least doubly transitive and hence primitive); and thus, by the induction hypothesis, $G$ is $N_{0}+(r+1)=(N+r)$-fold transitive on $X$.

Consider all the sets $(Y) g, g \in G$. If for every $g_{1}, g_{2} \in G,(Y) g_{1}$ $\cap(Y) g_{2}=\varnothing$ or $(Y) g_{1}=(Y) g_{2}$, then the family of sets $\{(Y) g: g \in G\}$ (by the transitivity of $G$ on $X$ and the fact $Y \neq X$ and $|Y| \geqq 2$ ) would constitute imprimitivity sets of $G$ on $X$, a contradiction. Hence there exist $g_{1}, g_{2} \in G$ such that $(Y) g_{1} \cap(Y) g_{2} \neq \varnothing$ and $(Y) g_{1} \neq(Y) g_{2}$. Thus $Y \cap(Y)\left(g_{2} g_{1}^{-1}\right) \neq \varnothing$ and $Y \neq(Y)\left(g_{2} g_{1}^{-1}\right)$, giving a $g_{3} \in G$ such that $Y \cap(Y) g_{3} \neq \varnothing$ and $Y \neq(Y) g_{3}$. Let $C_{3}=Y \cap(Y) g_{3}, A_{3}=Y \backslash C_{3}$ and $B_{3}=(Y) g_{3} \backslash C_{3}$. One of the sets $A_{3}, B_{3}$ is nonvoid. Let $s_{3}=\left|A_{3}\right|$. By Proposition 4, we have (i) $Y=C_{3} \cup A_{3},(Y) g_{3}=C_{3} \cup B_{3}$, (ii) $A_{3}, B_{3}$ and $C_{3}$ are pairwise disjoint and (iii) $s_{3}=\left|B_{3}\right|$ and $s_{3}$ is finite. Hence $A_{3}$, $B_{3}$ and $C_{3}$ are all nonvoid, $\left|X \backslash\left[Y \cup(Y) g_{3}\right]\right|=\left|(X \backslash Y) \backslash B_{3}\right|=|X \backslash Y|$ $-\left|B_{3}\right|=N-s_{3}$, and $s_{3} \geqq 1$.

Choose $g_{0} \in G$ such that $Y \cap(Y) g_{0} \neq \varnothing, \quad Y \neq(Y) g_{0}$ and $s_{0}=\left|Y \backslash\left[Y \cap(Y) g_{0}\right]\right|$ is minimal $\left(s_{0} \geqq 1\right)$. Let $C_{0}=Y \cap(Y) g_{0}$, $A_{0}=Y \backslash C_{0}$, and $B_{0}=(Y) g_{0} \backslash C_{0}$. By Proposition 2, $H_{0}=\left\{H, H^{0_{0}}\right\}$ is transitive on $Y_{0}=Y \cup(Y) g_{0}=Y \cup B_{0}$ and fixes $X \backslash Y_{0}$. Suppose that $s_{0}=1$. Since $H \leqq H_{0}$ is $r$-fold transitive on $Y \subseteq Y_{0}$ and fixes $Y_{0} \backslash Y=B_{0}$, then (by Proposition 3 with $\left.s=s_{0}=1\right) H_{0}$ is $(r+1)$-fold transitive on 
$Y_{0}$ and fixes $X \backslash Y_{0}=(X \backslash Y) \backslash B_{0}$, where $\left|X \backslash Y_{0}\right|=N-s_{0}=N_{0}$. Furthermore, since $Y \subseteq Y_{0},\left|Y_{0}\right| \geqq 2$. That is, if $s_{0}=1$ then $G$ is $(N+r)$-fold transitive on $X$.

In fact, $s_{0}=1$. For, suppose, that $s_{0}>1 .(Y) g_{0}=C_{0} \cup B_{0}$, where $B_{0}=\left\{b_{1}, \cdots, b_{s_{0}}\right\}$ and $C_{0} \neq \varnothing$. Since $H^{o_{0}}$ is primitive on $(Y) g_{0}$ and $\left|B_{0}\right|=s_{0} \geqq 2$, there is an element $h^{1} \in H^{g_{0}}$ which maps one or more (but not all) of the elements of $B_{0}$ onto elements of $B_{0}$ and the remaining elements of $B_{0}$ onto elements of $C_{0}$ : i.e., by renumbering if necessary, $b_{1}, \cdots, b_{u}$ (where $1 \leqq u<s_{0}$ ) are mapped onto elements of $B_{0}$, and $b_{u+1}, \cdots, b_{s_{0}}$ are mapped onto elements of $C_{0}$ by $h^{1}$. Since $h^{1}$ maps $(Y) g_{0}$ onto itself and $B_{0}$ is finite, precisely $\left(s_{0}-u\right)$ elements of $B_{0}$ are the images of $\left(s_{0}-u\right)$ elements of $C_{0}$, say $c_{1}, \cdots, c_{u_{0}-u}$. Hence the elements of $C_{0} \backslash\left\{c_{1}, \cdots, c_{s_{0}-u}\right\}$ are mapped by $h^{1}$ onto $C_{0} \backslash\left(\left\{b_{u+1}, \cdots, b_{s_{0}}\right\}\right) h^{1} \subseteq C_{0}$. Since $h^{1}$ fixes $A_{0}\left(A_{0} \subseteq X \backslash(Y) g_{0}\right)$, we have

$$
\begin{aligned}
(Y) h^{1} & =\left(C_{0} \cup A_{0}\right) h^{1}=A_{0} \cup\left(C_{0}\right) h^{1} \\
& =A_{0} \cup\left(C_{0} \backslash\left\{c_{1}, \cdots, c_{s_{0}-u}\right\}\right) h^{1} \cup\left(\left\{c_{1}, \cdots, c_{s_{0}-u}\right\}\right) h^{1} \\
& =A_{0} \cup\left[C_{0} \backslash\left(\left\{b_{u+1}, \cdots, b_{s_{0}}\right\}\right) h^{1}\right] \cup\left(\left\{c_{1}, \cdots, c_{s_{0}-u}\right\}\right) h^{1} .
\end{aligned}
$$

But since $Y \cap\left(\left\{c_{1}, \cdots, c_{s_{0}-u}\right\}\right) h^{1}=\varnothing$, then

$$
Y \cap(Y) h^{1}=\left(A_{0} \cup C_{0}\right) \backslash\left(\left\{b_{u+1}, \cdots, b_{s_{0}}\right\}\right) h^{1} \neq \varnothing .
$$

But $(Y) h^{1} \neq Y$ and $Y \backslash\left[Y \cap(Y) h^{1}\right]=\left(\left\{b_{u+1}, \cdots, b_{s_{0}}\right\}\right) h^{1}$ which has cardinal number $s_{0}-u<s_{0}$. This contradicts the minimality of $s_{0}$, and, hence, $s_{0}=1$.

Proof of Theorem 2. We wish to show that there is a subgroup $K_{0} \leqq G$ which has the properties: (i) $K_{0}$ is transitive on some subset $Y_{K_{0}}$ of $X$, (ii) $K_{0}$ fixes $X \backslash Y_{K_{0}}$ and (iii) $\left|X \backslash Y_{K_{0}}\right|=1$. Then Theorem 2 will follow from Proposition 3.

Let $\Re$ be the collection of all subgroups $K \leqq G$ such that: (i) $K$ is transitive on some subset $Y_{K} \subseteq X$, (ii) $K$ fixes the elements of $X \backslash Y_{K}$, (iii) $\left|Y_{K}\right| \geqq 2$ and (iv) $X \backslash Y_{K}$ is a finite, nonvoid set. Clearly $H \in \mathfrak{K}$. Furthermore there is a subgroup $K_{0} \in \mathcal{K}$ such that $\left|X \backslash Y_{K_{0}}\right| \leqq\left|X \backslash Y_{K}\right|$ for every $K \in \mathcal{K}$. To complete the proof we now show that $\left|X \backslash Y_{K_{0}}\right|=1$.

Since $G$ is primitive, by the same argument that was used in the proof of Theorem 1, there exists $g_{0} \in G$ such that $Y_{K_{0}} \neq\left(Y_{K_{0}}\right) g_{0}$ and $Y_{K_{0}} \cap\left(Y_{K_{0}}\right) g_{0} \neq \varnothing$. Also, if $C_{0}=Y_{K_{0}} \cap\left(Y_{K_{0}}\right) g_{0}, A_{0}=Y_{K_{0}} \backslash C_{0}$, and $B_{0}=\left(Y_{K_{0}}\right) g_{0} \backslash C_{0}$, then (i) $Y_{K_{0}}=C_{0} \cup A_{0}$, $\left(Y_{K_{0}}\right) g_{0}=C_{0} \cup B_{0}$, (ii) $A_{0}, B_{0}$ and $C_{0}$ are pairwise disjoint, (iii) $\left|A_{0}\right|=\left|B_{0}\right| \leqq\left|X \backslash Y_{K_{0}}\right|$, (iv) $A_{0}, B_{0}$, $C_{0}$ are all nonvoid, and (v) $\left|X \backslash\left[Y_{K_{0}} \cup\left(Y_{K_{0}}\right) g_{0}\right]\right|=\left|X \backslash Y_{K_{0}}\right|-r$, where $r=\left|A_{0}\right| \geqq 1$ and is finite. 
By Proposition 2, $\left\{K_{0}, K_{0}^{0_{0}}\right\}$ is transitive on $W=Y_{\mathbf{K}_{0}} \cup\left(Y_{\boldsymbol{K}_{0}}\right) g_{0}$ (hence $|W| \geqq 2$ ) and fixes the elements of $X \backslash W$. But $|X \backslash W|$ $=\left|X \backslash Y_{K_{0}}\right|-r<\left|X \backslash Y_{K_{0}}\right|$. Hence (by the minimality of $\left|X \backslash Y_{K_{0}}\right|$ ) it must be that $X \backslash W=\varnothing$, i.e., $X=W$.

We can write $A_{0}=\left\{a_{1}, \cdots, a_{r}\right\}, B_{0}=\left\{b_{1}, \cdots, b_{r}\right\}, Y_{K_{0}}=C_{0}$ $\cup\left\{a_{1}, \cdots, a_{r}\right\}, \quad\left(Y_{K_{0}}\right) g_{0}=C_{0} \cup\left\{b_{1}, \cdots, b_{r}\right\}$, and $X=C_{0}$ $\cup\left\{a_{1}, \ldots, a_{r}\right\} \cup\left\{b_{1}, \cdots, b_{r}\right\}$. We also observe that $\left|Y_{K_{0}}\right|>r$ $=\left|X \backslash Y_{K_{0}}\right|$.

Suppose $r>1$, i.e., $\left|X \backslash Y_{K_{0}}\right|>1$. Then since $G$ is primitive there is some $g \in G$ which maps some " $b$ " to a " $b$," say $b_{i}$ to $b_{j}$, and some " $b$ " to an " $a$ " or " $c$ " $\left(c \in C_{0}\right)$. Since the number of " $b$ "s is finite, not all " $b$ "s can be images of " $b$ "s under $g$. Hence $g$ must map some " $a$ " or " $c$ " to a " $b, "$ say to $b_{k}$. Further, since $r<\left|Y_{K_{0}}\right|$, not every " $a$ " and " $c$ " can be mapped onto a " $b$ " by $g$. Therefore some " $a$ " or " $c$ " is mapped by $g$ onto an " $a$ " or " $c$," i.e., $Y_{K_{0}} \cap\left(Y_{K_{0}}\right) g \neq \varnothing$; furthermore, $b_{k} \in\left(Y_{K_{0}}\right) g \backslash Y_{K_{0}}$ and $b_{j} \notin Y_{K_{0}} \cup\left(Y_{K_{0}}\right) g=V$. Hence $Y_{K_{0}} \neq V,|V| \geqq 2$ and $0 \neq|X \backslash V|<\left|X \backslash Y_{K_{0}}\right|$. But by Proposition 2, $\left\{K_{0}, K_{0}^{0}\right\}$ is transitive on $V$ and fixes $X \backslash V$. Hence, by the minimality of $\left|X \backslash Y_{K_{0}}\right|$, $\left|X \backslash Y_{K_{0}}\right| \leqq|X \backslash V|$, a contradiction, so that $\left|X \backslash Y_{K_{0}}\right|=1$, and $G$ is doubly transitive on $X$.

\section{REFERENCE}

1. Marshall Hall, Jr., The theory of groups, Macmillan, New York, 1961.

WASHINGTON UNIVERSITY 